\title{
Rectification et addition à ma note "Sur l'unicité du développement trigonométrique“.
}

$$
\text { Par. }
$$
Alexandre Rajchman (Varsovio).

1. Rectification. Dans ma note "Sur l'unicité du développement trigonométrique ${ }^{\prime}$ (Fiundamenta Mathematicae, vol. III, p. 287, suiv.) se sont glissées quelques erreurs typographiques et meme à la fin (démonstration du théorème de M. Steinhaus) un lapsus plas grave, quoique sans conséquence.

Tout sera arrangé, si l'on prendra le soin de faire au volume IUI de ${ }_{n}$ Fundamenta " des rectifications suivantes

page 294: l'inégalité (11) doit étre écrite: $\sqrt{a_{n}^{2}+b_{n}^{2}} \leqq \eta$.

page 295: 5 me ligne d'en bas doit étre écrite: $A=\alpha_{k}+\frac{\delta}{8} ; B=\beta_{k}-\frac{\delta}{8}$. page 301: les deux lignes qui suivent l'inégalité (28) doivent être rayées; les deux lignes suivantes sont à écrire comme suit:

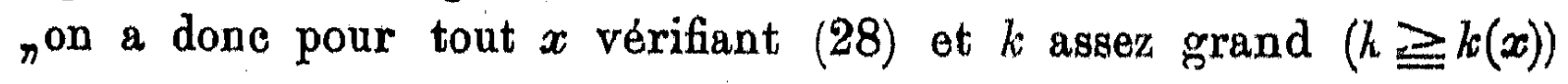

$$
\left|\cos 2 \pi n_{k}\left(x-h_{n_{k}}\right)\right| \leqq 1-\frac{1}{2_{p}}{ }^{\mu}
$$

Dans les lignes suivantes jusqu'à la fin de la page on doit lire ${ }_{n}\left(29_{k}\right)^{\mu}$ (au lieu $\left(29_{j}\right)$ ); $n^{p}\left(k_{i}\right)^{\mu}$ (au lieu de $Z^{p}$ ) et ${ }_{n} d_{n_{k}}{ }^{\mu}$ (au lieu de $\left.d_{k}\right)$.

L. la fin de la page 301 on doit ajouter la phrase suivante: non voit de meme que les ensembles

$$
Z_{q}^{p}=z^{p}(q) z^{p}(q+1) \ldots \quad(q=2,3 \ldots)
$$

sont tous du type $(H)$. 
Enfin: page 302, 2-me ligne d'en haut doit être écrite comme il suit:

$$
E^{p} \subset Z_{1}^{p}+Z_{2}^{p}+\ldots+Z_{q}^{p}+\ldots
$$

2. Résultats de M $^{\text {lle }}$ Bary. Le fait de l'existence des ensembles

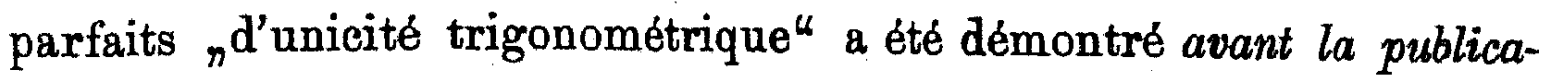
tion de ma note, en 1921, par $\mathbf{M}^{110}$ Nina Bary au Séminaire Mathématique de l'Université de Moscou, dirigé par prof. N. L u sin ${ }^{1}$ ). Par conséquent, le nouveau de ma note ce n'est pas l'existence d'une classe d'ensembles parfaits "d'unicité trigonométrique ${ }^{u}$, mais le fait que tout ensemble "du type $(B)^{4}$ (et, en particulier, "l'ensemble de Cantor") est un ensemble d'unicité. M Me Bary vient de généraliser ce résultat en prouvant que non seulement les ${ }_{n} H^{4}$, mais les ${ }_{n} H_{\sigma}{ }{ }^{2}$ (c'est-à-dire des ensembles formés par la réunion d'une infinité dénombrable des ensembles du type $H$ ) sont des nensembles d'unicité"

Ce résultat gagne de l'importence si l'on remarque que l'ensemble de points où l'on a

$$
\limsup _{n \rightarrow \infty}\left|a_{n} \cos n x+b_{n} \sin n x\right| \neq \limsup _{n \rightarrow \infty} \sqrt{a_{n}^{2}+b_{n}^{2}}
$$

est aussi $H_{\sigma}$ (ou sous-ensemble d'un $H_{\sigma}$ ) (Cf. la fin de ma note, Fund. Math. vol. III pp. 299-301).

C'est done le même type d'ensembles qui intervient dans la solution de deux problèmes trigonométriques de Cantor: du problème de la limite de $\left|a_{n} \cos n x+b_{n} \sin n x\right|$ et du problème de l'anicité du développement trigonométrique.

C'est à l'obligeance de $M^{\text {le }}$ Bary et du prof. Lusin que je dois la connaissance de ce résultat; le mémoire de $\mathrm{M}^{\mathrm{He}} \mathrm{Bary}$ paraîtra probablement dans le $\mathrm{V}^{\mathrm{me}}$ vol. des ${ }_{n}$ Fundamenta .

1) Cette démonstration n'a pas été publiée. 